\title{
Transtornos alimentares em alunas de nutrição do Rio Grande do Sul
}

\author{
Eating disorders in female nutrition students \\ in Rio Grande do Sul state, Brazil
}

Vanessa Ramos KIRSTEN ${ }^{1}$

Fernanda FRATTON²

Nádia Behr Dalla PORTA ${ }^{3}$

RES U M O

\section{Objetivo}

Verificar sintomas de transtornos alimentares em estudantes de Nutrição do sexo feminino em relação ao estado nutricional, à prática de atividade física e ao semestre cursado.

\section{Métodos}

Foi utilizado o Eating Attitudes Test-26 para verificar sintomas de transtornos alimentares em estudantes do curso de Nutrição de uma faculdade particular do Rio Grande do Sul. Foram coletados dados de peso e altura para a avaliação do estado nutricional, semestre cursado, prática de atividade física e dados demográficos.

\section{Resultados}

A amostra foi composta por 186 alunas. As entrevistadas, em sua maioria, apresentavam entre 21 e 25 anos de idade (50,0\%), cor branca (93,5\%), renda inferior a 10 salários-mínimos (66,0\%) e eram solteiras $(91,0 \%)$. Os resultados do Eating Attitudes Test-26 demonstraram que $24,7 \%$ das estudantes apresentavam sintomas de transtornos alimentares. Cerca de $85,5 \%$ das estudantes da amostra eram eutróficas, 8,5\% apresentavam algum grau de desnutrição e $6,0 \%$, sobrepeso e/ou obesidade. Não houve significância estatística na comparação entre sintomas de transtornos alimentares com o estado nutricional $(p=0,32)$, o semestre cursado $(p=0,67)$ e com a prática de atividade física $(p=0,12)$.

\section{Conclusão}

Foi identificada alta proporção de sintomas de transtornos alimentares, porém sem significância estatística quando comparado com o estado nutricional, à atividade física e ao semestre cursado.

Termos de indexação: Estado nutricional. Estudantes de ciências da saúde. Questionários. Transtornos alimentares.

\footnotetext{
1 Centro Universitário Franciscano, Curso de Nutrição. R. dos Andradas, 1614, Centro, 97010-032, Santa Maria, RS, Brasil Correspondência para/Correspondence to: V.R. KIRSTEN. E-mails: <vanessark@unifra.br; vanessa_kirsten@yahoo.com.br>.

2 Nutricionista. Santa Maria, RS, Brasil.

3 Nutricionista. Florianópolis, SC, Brasil.
} 


\section{A B S T R A C T}

\section{Objective}

The objective of this study was to check for symptoms of eating disorders in female nutrition students in relation to their nutritional status, level of physical activity and academic semester.

\section{Methods}

The Eating Attitudes Test-26 was used to check for symptoms of eating disorders in nutrition students of a private college in Rio Grande do Sul, Brazil. The following data were collected: weight, height, academic semester, level of physical activity and demographic data.

\section{Results}

The sample consisted of 186 female students. Most of the interviewees aged between 21 and 25 years (50.0\%), were Caucasian (93.5\%), single (91.0\%) and made less than US\$2080 per month (66.0\%). The Eating Attitudes Test-26 results showed that $24.7 \%$ of these students had symptoms of eating disorders. Around $85.5 \%$ of the interviewees had normal weight, $8.5 \%$ had some degree of malnutrition and $6.0 \%$ were overweight and/or obese. No statistical significance was found when the symptoms of eating disorders were compared with nutritional status $(p=0.32)$, academic semester $(p=0.67)$ and level of physical activity $(p=0.12)$.

\section{Conclusion}

There is a high prevalence of symptoms of eating disorders in this sample, but they are not statistically significance with nutritional status, level of physical activity and academic semester.

Indexing terms: Nutritional status. Students health occupations. Eating disorders. Questionnaires.

\section{N T R O D U ÇÃ O}

Atualmente, tem-se discutido a influência dos fatores socioculturais que impõem um ideal de beleza associado ao culto às dietas com restrição de energia e ao corpo esquálido, como gatilhos no desenvolvimento dos transtornos alimentares ${ }^{1-3}$.

O medo obsessivo da obesidade faz com que cada vez mais mulheres controlem o peso corporal, com o uso de dietas milagrosas, exercícios exagerados, laxantes, diuréticos e drogas anorexígenas. O gênero feminino, geralmente, é o mais vulnerável à aceitação das pressões sociais, econômicas e culturais associadas aos padrões estéticos e, por isso, mais suscetível aos transtornos alimentares, representando $95 \%$ dos casos $^{1,4}$. As formas mais descritas na literatura são: a anorexia nervosa (AN), a bulimia nervosa (BN) e o transtorno de compulsão alimentar periódica $(\mathrm{TCAP})^{5}$.

A anorexia nervosa é caracterizada por uma perda de peso auto-imposta, acompanhada de disfunção endócrina e atitude psicopatológica distorcida em relação à imagem, à alimentação e ao peso ${ }^{6}$. Na BN, as características essenciais consistem em episódios de compulsão alimentar e métodos compensatórios inadequados para evitar o ganho de peso. Para fins diagnósticos, a compulsão e os comportamentos compensatórios inadequados devem ocorrer, em média, pelo menos duas vezes por semana há três meses? ${ }^{7}$.

A prevalência de bulimia nervosa clinicamente significativa em estudantes universitárias é muito maior do que a observada na sociedade, correspondendo a, aproximadamente, 4,0\% ${ }^{8-10}$. A AN é considerada rara, visto que a prevalência em mulheres jovens é de $0,5 \%{ }^{11}$.

Ainda existe muita controvérsia sobre os métodos mais adequados para a avaliação desses transtornos. Os instrumentos para a avaliação dos transtornos alimentares (TA) surgiram com a necessidade de sistematizar os estudos a partir do estabelecimento e do aprimoramento de seus critérios diagnósticos. De um modo geral, os instrumentos de avaliação são agrupados em, pelo menos, três categorias: 1) questionários autoaplicáveis; 2) entrevistas clínicas e 3) automonitoração ${ }^{12}$. 
O Eating Attitudes Test (EAT) ou Teste de Atitudes Alimentares, desenvolvido por Garner \& Garfinkel ${ }^{13}$, é um dos instrumentos mais utilizados atualmente em estudos com anorexia nervosa, com o objetivo de medir sintomas da síndrome de maneira mais fácil e rápida, favorecendo, assim, a precocidade do diagnóstico e do tratamento, evitando a evolução da doença.

Dentre os fatores precipitantes na etiologia dos transtornos alimentares, a dieta para emagrecer é o fator mais freqüente ${ }^{14}$. Nesse contexto tem-se questionado se futuros nutricionistas que, na sua grande maioria, são formados por mulheres jovens, por estarem constantemente preocupados com a imagem corporal, com o sobrepeso e com a alimentação, podem ser mais suscetíveis ao desenvolvimento de transtornos alimentares. Frente a essa questão, o objetivo deste estudo foi verificar sintomas de transtornos alimentares em acadêmicas de Nutrição, em relação ao estado nutricional, a dados demográficos e socioeconômicos, ao semestre cursado e à prática de atividade física.

\section{MÉTODOS}

O estudo foi realizado após aprovação do Comitê de Ética do Centro Universitário Franciscano cujos registros são: CEP/UNIFRA: 014.2006.02 e CONEP:1246.

O delineamento do estudo foi caracterizado como transversal, em que as participantes foram constituídas por amostra de conveniência, com estudantes do curso de Nutrição do sexo feminino de uma faculdade particular do centro do estado do Rio Grande do Sul, que aceitaram participar da pesquisa durante os meses de abril e maio de 2006, assinando o Termo de Consentimento Livre Esclarecido, conforme Resolução 196/96 15 .

Os pesquisadores apresentavam-se ao professor que estivesse na sala de aula e solicitavam 15 minutos para a aplicação do questionário. Este era respondido individualmente, após breve explicação sobre o trabalho, no qual foram expostos os objetivos da pesquisa.

Os critérios de exclusão foram: 1) fornecer questionários incompletos; 2) ser do sexo masculino. A escolha pelo sexo feminino foi devido à maior prevalência de transtornos alimentares nesse grupo, pois, em publicação prévia, na qual foram revisadas 17 pesquisas sobre bulimia nervosa, as prevalências variaram de 5 a $20 \%$ nas mulheres e, de 0 a $5 \%$ nos homens ${ }^{16}$. As perdas foram: 1) estudantes ausentes no dia em que foi aplicado o questionário; 2) estudantes que não assinaram o Termo de Consentimento Livre e Esclarecido.

O questionário, de autopreenchimento, não continha identificação pessoal dos participantes. Foi realizado controle sobre o número de alunas matriculadas $(n=280)$, o número de ausentes no dia e de questionários incompletos $(n=94)$ e o número de questionários recebidos completos $(n=186)$.

Coletaram-se os seguintes dados:

- Peso (em kg), por meio de balança analógica com capacidade máxima de $130 \mathrm{~kg}$ e precisão de $0,1 \mathrm{~kg}$. As estudantes foram pesadas descalças e com o mínimo de roupa possível. A altura foi verificada com o uso de fita métrica inextensível, da marca Cescorf, fixada à parede sem rodapés. A classificação do estado nutricional foi calculada pelo Índice de Massa Corporal (IMC), que é resultante do peso dividido pela altura $\left(\mathrm{kg} / \mathrm{m}^{2}\right)$, classificado segundo Organização Mundial da Saúde ${ }^{17}$.

- Dados de idade, cor, atividade física (tipo de atividade e freqüência na semana), renda familiar, semestre cursado serviram para compor o perfil demográfico e socioeconômico da amostra com questões fechadas pertencentes a um questionário não validado elaborado pelos autores desta pesquisa, baseado em informações de outras publicações. A parte do questionário que abordava a atividade física apenas questionava se a mesma era realizada regularmente e quantas vezes na semana. 
- Questionário sobre sintomas de TA (Eating Attitude Test, EAT-26), respondido pelas estudantes. Esse questionário foi traduzido e validado para a língua portuguesa, mantendo correlações clínicas e psicométricas entre grupos de pacientes e sujeitos normais ${ }^{18}$. O ponto de corte estabelecido foi de 21 pontos. Acima desse valor, os participantes são classificados como grupo de risco ao desenvolvimento do transtorno alimentar. Cada questão apresentou seis opções de resposta, conferindo-se pontos de 0 a 3 , dependendo da escolha (sempre $=3$ pontos, muitas vezes $=2$ pontos, às vezes $=1$ ponto, poucas vezes $=0$ ponto, quase nunca $=0$ ponto e nunca $=0$ ponto). A única questão que apresentou pontos em ordem invertida foi a 25; para respostas mais sintomáticas, como o sempre, muitas vezes e às vezes não são dados pontos, e para as alternativas poucas vezes, quase nunca e nunca são conferidos 1, 2 e 3 pontos, respectivamente ${ }^{13}$.

Para a análise dos dados, foi utilizado o software Epi Info, versão 6.0, para o cálculo de medidas descritivas e de comparação das características (estado nutricional, semestre cursado e prática de atividade física) entre a amostra considerada com sintomas de TA (EAT +: pontuação maior que 21 pontos) e sem sintomas de TA (EAT -: pontuação menor que 21 pontos) por meio do teste Qui-quadrado $\left(\chi^{2}\right)$ para tendência (Chi-Square for trends). Consideraram-se significativos os valores que apresentaram $p<0,05$ e intervalo de confiança de $95 \%$.
RES U LTA D OS

Foram avaliadas 186 alunas do $1^{\circ}$ ao $8^{\circ}$ semestre do curso de Nutrição de uma faculdade particular do Centro do Estado do Rio Grande do Sul. Verificou-se que as entrevistadas, em sua maioria, apresentavam entre 21 e 25 anos de idade $(50,0 \%)$, cor branca $(93,5 \%)$, renda inferior a 10 salários-mínimos $(66,0 \%)$ e eram solteiras $(91,0 \%)$ (Tabela 1).

Tabela 1. Características demográficas e socioeconômicas de estudantes de nutrição do sexo feminino $(n=186)$. Santa Maria (RS), 2006.

\begin{tabular}{lcc}
\hline \multirow{2}{*}{ Faixa etária } & \multicolumn{2}{c}{ Estudantes } \\
\cline { 2 - 3 } & $\mathrm{n}$ & $\%$ \\
\hline Idade (em anos) & 69 & 38,0 \\
17 - 20 & 94 & 50,0 \\
$21-25$ & 18 & 10,0 \\
$26-30$ & 5 & 2,0 \\
31 - 41 & & \\
Cor & 174 & 93,5 \\
Branca & 1 & 0,5 \\
Negra & 5 & 3,0 \\
Mulata & 6 & 3,0 \\
Amarela & & \\
Renda familiar (em salários-mínimos) & & \\
1 até 5 & 41 & 22,0 \\
5 até 10 & 64 & 34,0 \\
10 até 15 & 46 & 25,0 \\
15 até 20 & 22 & 12,0 \\
Acima de 20 & 13 & 7,0 \\
Estado civil & & \\
Solteira & & \\
Casada & 170 & 91,0 \\
Divorciada & 13 & 7,0 \\
\hline Total & 3 & 2,0 \\
\hline & 186 & 100,0 \\
\hline & &
\end{tabular}

Tabela 2. Estado nutricional das estudantes de Nutrição do sexo feminino com e sem sintomas de transtornos alimentares. $(p=0,32)$. Santa Maria (RS), 2006

\begin{tabular}{|c|c|c|c|c|c|c|}
\hline \multirow{2}{*}{ Estado nutricional } & \multicolumn{2}{|c|}{ EAT + } & \multicolumn{2}{|c|}{ EAT - } & \multicolumn{2}{|c|}{ Total } \\
\hline & $\mathrm{n}$ & $\%$ & $\mathrm{n}$ & $\%$ & $n$ & $\%$ \\
\hline Desnutrição & 2 & 12,5 & 14 & 87,5 & 16 & 100,0 \\
\hline Eutrofia & 41 & 25,8 & 118 & 74,2 & 159 & 100,0 \\
\hline Sobrepeso e obesidade & 3 & 27,3 & 8 & 72,7 & 11 & 100,0 \\
\hline Total & 46 & 24,7 & 140 & 75,3 & 186 & 100,0 \\
\hline
\end{tabular}

EAT +: com sintomas de transtorno alimentar; EAT - sem sintomas de TA; EAT: Eating attitudes test; TA: transtornos alimentares; \%: porcentagem; n: número da amostra. 
Tabela 3. Semestre cursado por estudantes de Nutrição do sexo feminino com e sem sintomas de transtornos alimentares. $(p=0,67)$. Santa Maria (RS), 2006.

\begin{tabular}{|c|c|c|c|c|c|c|}
\hline \multirow{2}{*}{ Semestre } & \multicolumn{2}{|c|}{ EAT + } & \multicolumn{2}{|c|}{ EAT - } & \multicolumn{2}{|c|}{ Total } \\
\hline & $n$ & $\%$ & $n$ & $\%$ & $n$ & $\%$ \\
\hline $1^{\circ}$ & 8 & 29,6 & 19 & 70,4 & 27 & 100,0 \\
\hline $2^{\circ}$ & 3 & 12,5 & 21 & 87,5 & 24 & 100,0 \\
\hline $3^{\circ}$ & 4 & 33,3 & 8 & 66,7 & 12 & 100,0 \\
\hline $4^{\circ}$ & 5 & 27,8 & 13 & 72,2 & 18 & 100,0 \\
\hline $5^{\circ}$ & 9 & 31,0 & 20 & 69,0 & 29 & 100,0 \\
\hline $6^{\circ}$ & 7 & 33,4 & 14 & 66,6 & 21 & 100,0 \\
\hline $7^{\circ}$ & 3 & 15,0 & 17 & 85,0 & 20 & 100,0 \\
\hline $8^{\circ}$ & 3 & 20,0 & 28 & 80,0 & 35 & 100,0 \\
\hline Total & 46 & 24,7 & 140 & 75,3 & 186 & 100,0 \\
\hline
\end{tabular}

EAT +: com sintomas de transtorno alimentar; EAT: sem sintomas de TA; EAT: Eating attitudes test; TA: transtornos alimentares; \%: porcentagem; n: número da amostra.

Quando analisados os sintomas para o desenvolvimento de TA pelo EAT-26, 24,7\% das estudantes de nutrição apresentaram sintomas de transtornos alimentares (EAT+).

O estado nutricional, avaliado pelo IMC, demonstrou que 85,5\% da amostra apresentaram eutrofia, 8,5\% risco de desnutrição e 6,0\% das alunas possuíam algum nível de sobrepeso e/ou obesidade.

Para a comparação do estado nutricional de estudantes de nutrição com (EAT +) e sem (EAT -) sintomas para transtornos alimentares, foi realizado o teste Qui-quadrado $\left(\chi^{2}\right)$. Não houve diferença entre estado nutricional e sintomatologia de TA $(p=0,32)$ (Tabela 2$)$.

Os sintomas de TA foram comparados entre as estudantes de cada semestre, conforme a Tabela 3. As alunas do $1^{\circ}$ semestre $(17,0 \%)$ e do $5^{\circ}$ semestre $(19,5 \%)$ apresentaram maiores proporções de EAT+. No entanto, não houve diferença estatisticamente significante $(p=0,67)$ entre os semestres.

Quando analisados a prática de atividade física das entrevistadas e os sintomas de TA, as estudantes que mais referiram realizar atividade física (quatro ou mais vezes na semana) apresentaram mais sintomas associados com TA $(37,0 \%)$, quando comparadas com as que realizam até três vezes na semana $(24,6 \%)$ e com as meninas que não realizam atividade física (21,3\%). No entanto esta diferença não apresentou significância estatística $(p=0,12)$ (Tabela 4$)$.

\section{DISCUSS Ã O}

De acordo com Morgan \& Claudino ${ }^{14}$, os transtornos alimentares são ainda hoje doenças raras que acometem principalmente adolescentes ou mulheres jovens. Acreditava-se que os transtornos alimentares limitavam-se a um grupo constituído por mulheres jovens, brancas, pertencentes à elite e residentes em países ricos, algo que vem sendo contestado pelo número crescente de relatos de transtornos alimentares em países em desenvolvimento e em diferentes etnias ${ }^{19}$.

Em um estudo de base populacional realizado no Sul do Brasil, que estudou a associação entre percepção corporal e comportamentos alimentares anormais em mulheres de 12 a 29 anos,

Tabela 4. Prática de atividade física das estudantes de Nutrição com e sem sintomas de transtornos alimentares. $(p=0,12)$. Santa Maria (RS), 2006.

\begin{tabular}{|c|c|c|c|c|c|c|}
\hline \multirow{2}{*}{$\begin{array}{l}\text { Atividade física durante } \\
\text { a semana }\end{array}$} & \multicolumn{2}{|c|}{ EAT + } & \multicolumn{2}{|c|}{ EAT - } & \multicolumn{2}{|c|}{ Total } \\
\hline & $\mathrm{n}$ & $\%$ & $n$ & $\%$ & $n$ & $\%$ \\
\hline Não realiza & 20 & 21,3 & 74 & 78,7 & 94 & 100,0 \\
\hline Até 3 vezes & 16 & 24,6 & 49 & 75,4 & 65 & 100,0 \\
\hline 4 a 7 vezes & 10 & 37,0 & 17 & 63,0 & 27 & 100,0 \\
\hline Total & 46 & 24,7 & 140 & 75,3 & 186 & 100,0 \\
\hline
\end{tabular}

EAT +: com sintomas de transtorno alimentar; EAT: sem sintomas de TA; EAT: Eating attitudes test; TA: transtornos alimentares; \%: porcentagem; n: número da amostra. 
verificou-se que $49,9 \%$ da amostra apresentavam idades entre 20 e 29 anos, 90,1\% apresentavam cor branca e a renda familiar mais freqüente foi acima de 10 salários-mínimos mensais ${ }^{20}$. Os dados se assemelham ao presente estudo, quando comparados com cor e idade. Quanto à renda familiar, os dados não são semelhantes, provavelmente devido ao fato de que a amostra do estudo de Nunes et al. ${ }^{20}$ foi de base populacional com mulheres entre 12 e 29 anos de idade.

Apesar de o EAT-26, em estudos populacionais, apresentar um baixo valor preditivo para a anorexia nervosa (entre 100 pontuadores altos apenas 19 eram casos de anorexia nervosa), o instrumento pode ser utilizado como um índice da gravidade das preocupações típicas de pacientes com transtorno alimentar, principalmente a intenção de emagrecer e o medo de ganhar peso $^{21}$. Dessa forma, pode-se apontar a necessidade de intervenção coletiva ou, nos casos mais sintomáticos, de encaminhamento aos cuidados de profissional especializado.

São raros os estudos sobre a prevalência de transtornos alimentares na população, principalmente grupos específicos, como estudantes de cursos que estejam relacionados à alimentação, à nutrição e aos cuidados com a composição corporal.

Segundo Fiates \& Salles 5 , estudantes de nutrição estão em contato constante com o alimento e acham que a boa aparência pode ser uma importante medida de valor pessoal rumo a uma profissão de sucesso. Além disso, possuem conhecimentos quantitativos a respeito dos alimentos que podem usar para se manter de acordo com os rígidos padrões estéticos vigentes. Esses fatores sugerem que futuras nutricionistas se inserem em um ambiente favorável ao desenvolvimento de transtorno alimentar.

Pertencer a grupos profissionais como atletas, bailarinas, modelos e nutricionistas reforça a demanda por um corpo muito magro, aumentando o risco de transtorno alimentar ${ }^{3,19}$. No entanto, ainda não está esclarecido se o ambiente teria uma influência desencadeante desses transtornos ou se essas pessoas já seriam predispostas a desenvolver transtorno alimentar e, por isso, a procuraram tais profissões.

Em um estudo realizado com alunas de Medicina da Faculdade do Ceará ${ }^{16}$, os resultados da aplicação do EAT-26 demonstraram que 5,5\% da amostra apresentaram alto risco para o desenvolvimento de transtorno alimentar. Fiates \& Sales ${ }^{5}$, quando analisaram estudantes de Nutrição do Estado de Santa Catarina, encontraram que 25,0\% da amostra apresentavam suscetibilidade a transtornos alimentares, utilizando também o EAT-26. Esse dado se assemelha ao encontrado no presente estudo, no qual $24,7 \%$ da amostra também apresentaram sintomas para o desenvolvimento de transtornos alimentares. Stipp \& Oliveira ${ }^{22}$ analisaram estudantes de Nutrição de uma universidade particular de São Paulo, com a mesma metodologia, e obtiveram $18,0 \%$ das alunas com escore maior que 21.

Souza et al. ${ }^{16}$ sugerem que estudantes de escolas particulares poderiam apresentar taxas ainda mais altas de escores no teste EAT-26, por pertencerem à categoria econômica mais favorável e, assim, apresentarem um fator de risco para o desenvolvimento de transtornos alimentares. No entanto, não foram avaliados ainda sintomas de transtornos alimentares em estudantes de cursos universitários pertencentes a categorias econômicas menos favorecidas, para concluir que, realmente, estudantes de poder aquisitivo maior são mais suscetíveis a transtornos alimentares.

É importante ressaltar que, quando o percentual encontrado for maior que $20 \%$ de estudantes com EAT +, ele é considerado preocupante, evidenciando a importância da investigação desses sintomas nas populações de risco ${ }^{5}$.

Quando são analisados os critérios diagnósticos para anorexia e bulimia nervosa da Classificação Internacional de Doenças (CID), mulheres com anorexia nervosa mantêm o peso corpóreo em, pelo menos, $15,0 \%$ abaixo do esperado ou o IMC menor que $17,5 \mathrm{~kg} / \mathrm{m}^{22}$. Souza et al. ${ }^{16} \mathrm{e}$ Nunes et al. ${ }^{21}$ obtiveram, em suas amostras de 
universitárias, 91,0\% e 82,0\% de eutrofia, respectivamente, resultados que se assemelham aos dados deste trabalho $(85,5 \%)$.

Em um estudo que analisou os fatores de risco para o desenvolvimento de distúrbios alimentares em universitárias do curso de Nutrição e de outros cursos da Universidade Federal de Santa Catarina, verificou-se que, nas estudantes em que o EAT-26 foi positivo, $86,0 \%$ delas eram eutróficas ${ }^{5}$. É importante destacar que, neste estudo, a maioria das entrevistadas, tanto suscetíveis como não-suscetíveis, apresentou eutrofia. Além disso, verificou-se um número maior de estudantes classificadas com algum grau de magreza na categoria de não-suscetíveis a transtornos alimentares, o que evidencia a não relação entre essas duas categorias.

Um fato que pode ser associado aos sintomas de transtorno alimentar é o semestre cursado, para verificar se, no decorrer do curso, existe um aumento de transtorno alimentar em mulheres jovens que estudam em cursos da área da saúde. No entanto, para verificar tal fato, existe a necessidade de estudos longitudinais, verificando casos novos de alunas com suscetibilidade para o desenvolvimento desse tipo de transtorno. Souza et al. ${ }^{16}$ avaliaram a associação entre o semestre cursado e a prevalência de EAT + em estudantes de um curso de Medicina. A predominância de alunas que exibiam maior probabilidade aos transtornos alimentares não aumentou com o avanço dos semestres, o que se assemelhou aos resultados do presente trabalho. A relação entre essas duas variáveis foi realizada pela incerteza de conhecer se o avanço dos semestres no curso, ocasionando um maior conhecimento sobre a alimentação e os cuidados para manutenção do peso corpóreo, poderia influenciar no desenvolvimento de transtornos alimentares.

Como os transtornos alimentares são multifatoriais, e apresentam gravidade e manifestações clínicas diferentes, a sua identificação precoce torna-se importante. Uma avaliação adequada deve incluir ingestão alimentar (quantidade e qualidade) e prática de atividade física ${ }^{5,22}$. Nesse aspecto, o hábito de freqüentar academias diariamente pode estar desvinculado dos efeitos benéficos dos exercícios físicos sobre a saúde. A prática de atividade física, nesse caso, seria realizada exclusivamente para resultar em perda de peso.

Pode-se observar ainda que, quando analisada a prática de atividade física, $57 \%$ das estudantes suscetíveis a transtornos alimentares (EAT+) e $47 \%$ das não suscetíveis realizavam algum tipo de atividade física durante a semana. Esses dados foram menos expressivos que os apresentados por Fiates \& Salles ${ }^{5}$, nos quais $75 \%$ das estudantes de Nutrição com EAT+ praticavam exercícios físicos com um único propósito de aumentar o gasto energético.

Algumas limitações podem ser mencionadas em relação ao presente trabalho:

- O número de sujeitos da amostra deveria ser, no mínimo, 10 vezes o número de itens do questionário (26 questões) ${ }^{18}$ e foram avaliadas 186 estudantes.

- Segundo Nunes et al. ${ }^{18}$, os resultados do EAT-26 devem ser vistos com cautela nos seguintes aspectos: a) é inapropriado pensar que um escore alto se traduz em diagnóstico de transtorno alimentar, b) o teste indica a presença de padrões alimentares anormais, mas não revela, em si, a possibilidade psicopatológica subjacente ao comportamento manifesto.

- O método utilizado para detecção de casos se baseou, exclusivamente, em questionários de autopreenchimento. Esse procedimento não se mostra suficiente para estabelecer um diagnóstico de transtorno alimentar, uma vez que várias perguntas podem ter sido interpretadas de forma equivocada pelas respondentes ${ }^{16}$. Dessa forma, preferiu-se utilizar o termo suscetibilidade, pois não é possível diagnosticar a doença.

- Além disso, à medida que aspectos multifatoriais são responsáveis pela etiopatogenia dos transtornos alimentares, uma avaliação adequada deveria incluir métodos de mensuração da inges- 
tão alimentar (quantidade e qualidade), da atividade física e de aspectos psicopatológicos como imagem corporal, episódios bulímicos e comportamentos purgativos ${ }^{21}$.

- Houve uma perda significativa da amostra (33\%) em relação ao número de alunas matriculadas, diminuindo a confiabilidade e representatividade dos dados.

Mesmo considerando que a forma ideal de atender aos objetivos deste trabalho seria a de um estudo longitudinal, no qual as mesmas alunas pudessem ser avaliadas ao início e ao final do curso, e que estes resultados não podem ser extrapolados para a população brasileira em geral24, é possível e útil a generalização para outras populações semelhantes.

\section{O N CLUSÃ O}

Os resultados mostraram uma proporção alta de sintomas associados aos transtornos alimentares em acadêmicas do curso de Nutrição. Não só no presente trabalho, mas em outros já citados neste artigo, verifica-se que mulheres jovens, estudantes de cursos da área da nutrição, estão inseridas em uma população de risco para esses transtornos. Não se sabe ao certo se essas estudantes chegam à universidade com tais sintomas, ou se eles são desenvolvidos ao longo do curso. Dessa forma, ressalta-se a necessidade de maiores investigações com este grupo de estudantes para melhor conhecimento das causas ligadas aos sintomas de transtornos alimentares e às prováveis conseqüências na sua formação e posteriormente na sua atuação profissional.

\section{$C O L A B O R A C ̧ \tilde{A} O$}

V.R. KIRSTEN orientou o projeto auxiliou na coleta de dados, na redação do artigo, na análise e na interpretação dos dados. F. FRATTON participou na coleta dos dados e na redação do artigo. N.B.D. PORTA participou na coleta dos dados e na redação do artigo.

\section{REFERÊ N CIAS}

1. Andrade A, Bosi MLM. Mídia e subjetividade: impacto no comportamento alimentar feminino. Rev Nutr. 2003; 16(1):117-25.

2. Salzano FT, Cordas TA. Hospital-dia (HD) para transtornos alimentares: revisão da literatura e primeiras impressões do HD do ambulatório de bulimia e transtornos alimentares do IPqHCFMUSP (AMBULIM). Rev Psiqiatr Clín. 2003; 30(3):86-94.

3. Appolinário JC, Claudino AM. Transtornos alimentares. Rev Bras Psiquiatr. 2000; 22(Supl 2): 28-31.

4. Vilela JEM Lamounier JA, Dellaretti Filho MA, Barros neto JR, Horta GM. Transtornos alimentares em escolares. J Pediatr (Rio Janeiro). 2004; 80(1): 49-54.

5. Fiates GMR, Salles RK. Fatores de risco para o desenvolvimento de distúrbios alimentares: um estudo em universitárias. Rev Nutr. 2001; 14(Supl): 3-6.

6. Huse DM, Lucas AR. Transtornos comportamentais que afetam a ingestão de alimentos: anorexia nervosa, bulimia nervosa, e outras condições psiquiátricas. In: Shils ME, Olson JA, Shike M, Ross AC. Tratado de nutrição moderna na saúde e na doença. Barueri: Manole; 2003.

7. Manual diagnóstico e estatístico de transtornos mentais. Porto Alegre: Artmed; 2002.

8. Pyle RL, Halvorson PA, Newman PA, Golff GM. The Incidence of bulimia in Freshman College Students. Int J Eat Disord.1983; 2:75-85.

9. Katzman M, Wolchik S, Braver T. The Prevalence of frequent binge eating and bulimia in a non-clinical college sample. Int J Eat Disord. 1984; (3):53-62.

10. Drewnowski A, Yee DK, Krahn DD. Bulimia in college women: incidence and recovery rates. Am J Psychiatry. 1988; 145(6):753-5.

11. Aalto-Setala T, Marttunen M, Tuulio-Menrikson A, Poikolainen K, Lonnqvist J. One-month prevalence of depression and other DSM-IV disorders among young adults. Psychol Med. 2001; 31:791-801.

12. Freitas S, Govenstein C, Appolinase JC. Instrumentos para avaliação dos transtornos alimentares. Rev Bras Psiquiatr. 2002; 24(Supl 3):34-8.

13. Bighetti F. Tradução e validação do eating attitudes test (EAT-26) em adolescentes do sexo feminino na cidade de Ribeirão Preto SP [dissertação]. São Paulo: Universidade de São Paulo; 2003.

14. Morgan CM, Claudino AM. Epidemiologia e etiologia. In: Claudino AM, Zanella MT. Transtornos alimentares e obesidade. Barueri: Manole; 2005. 
15. Brasil. Ministério da Saúde. Conselho Nacional de Saúde. Resolução N 196. Brasília; 1996.

16. Souza FGM, Martins MCR, Montiero FCC, Menezes Neto GC, Ribeiro IB. Anorexia e bulimia nervosa em alunas da faculdade de medicina da Universidade Federal do Ceará UFC. Rev Psiquiatr Clin (São Paulo). 2002; 29(4):172-80.

17. World Health Organization. Physical status: the use and interpretation of anthropometry. Geneva: WHO; 1995. Report of a WHO Expert Committee.

18. Nunes MA, Bagatini LF, Atuchaim AL, Kunza, Ramos $D$, Silva JA. Distúrbios da conduta alimentar: considerações sobre o teste de atitudes alimentares (EAT). Revista ABP-APAL. 1994; 16(1):7-10.

19. Morgan CM, Vecchitti IR, Negrão AB. Etiologia dos transtornos biológicos, psicológicos e sócio-culturais. Rev Bras Psiquiatr. 2002; 24(Supl 3):18-23.

20. Nunes MA, Olinto MTA, Barros FC, Cauey S. Influência da percepção do peso e do índice de massa corporal nos comportamentos alimentares anormais. Rev Bras Psiquiatr. 2001; 23(1):46-7.

21. Cordás TA, Neves JEP. Escalas de avaliação de transtornos alimentares. Psiquiatr Clin. 1999; 26(1): 41-7.

22. Stipp LM, Oliveira MRM. Imagem corporal e atitudes alimentares: diferenças entre estudantes de nutrição e psicologia. Saúde Rev. 2003; 5(9): 47-51.

23. Claudino AM, Borges MBF. Classificação e diagnóstico. In: Claudino AM, Zanella MT. Transtornos alimentares e obesidade. Barueri: Manole; 2005.

24. Dunker KLL, Philippi ST. Hábitos e comportamentos alimentares de adolescentes com sintomas de anorexia nervosa. Rev Nutr. 2003; 16(1):51-60.

Recebido em: 14/5/2007

Versão final reapresentada em: 7/8/2008 Aprovado em: 9/9/2008 